\title{
Strain-specific copy number variation in the intelectin locus on the 129 mouse chromosome 1
}

\author{
Zen H Lu, Alex di Domenico, Steven H Wright, Pamela A Knight, C Bruce A Whitelaw, Alan D Pemberton*
}

\begin{abstract}
Background: C57BL/6J mice possess a single intelectin (It/n) gene on chromosome 1. The function of intelectins is not well understood, but roles have been postulated in insulin sensitivity, bacterial recognition, intestinal lactoferrin uptake and response to parasites and allergens. In contrast to C57BL/6J mice, there is evidence for expansion of the It/n locus in other strains and at least one additional mouse It/n gene product has been described. The aim of this study was to sequence and characterise the It/n locus in the 12957 strain, to determine the nature of the chromosomal expansion and to inform possible future gene deletion strategies.

Results: Six 12957 BAC clones were sequenced and assembled to generate $600 \mathrm{kbp}$ of chromosomal sequence, including the entire It/n locus of approximately $500 \mathrm{kbp}$. The locus contained six distinct It/n genes, two CD244 genes and several It/n- and CD244-related pseudogenes. It was approximately $433 \mathrm{kbp}$ larger than the corresponding C57BL/6J locus. The expansion of the It/n locus appears to have occurred through multiple duplications of a segment consisting of a full-length It/n gene, a CD244 (pseudo)gene and an It/n pseudogene fragment. Strong evidence for tissue-specific distribution of It/n variants was found, indicating that It/n duplication contributes more than a simple gene dosage effect.
\end{abstract}

Conclusions: We have characterised the It/n locus in 12957 mice to reveal six It/n genes with distinct sequence and expression characteristics. Since C57BL/6J mice possess only a single It/n gene, this is likely to contribute to functional differences between C57BL/6J and other mouse strains.

\section{Background}

Intelectins are glycoproteins with an approximate subunit size of $37 \mathrm{kDa}$, that have been described in mammals, fish and amphibians. The genome sequence of the sea squirt Ciona intestinalis [1] shows the presence of closelyrelated genes, indicating that intelectins arose early in chordate evolution. In mammals, intelectins have so far been described in humans [2-4], mice [5,6], sheep [7], cattle [8] and pigs [9], although interestingly, the dog genome [10] apparently lacks any intelectin genes.

The three-dimensional structures of intelectins have not yet been elucidated. Much of the predicted protein sequence is highly conserved across species, including eight cysteine residues and a fibrinogen-like domain [11]. There has been significant interest in the functions of intelectins, which appear to be many and varied. $\mathrm{A} \mathrm{Ca}^{2+}$-dependent galactose-specific lectin activity was

\footnotetext{
* Correspondence: alan.pemberton@ed.ac.uk

The Roslin Institute and Royal (Dick) School of Veterinary Sciences, University of Edinburgh, Roslin, Midlothian, UK
}

first detected in Xenopus intelectins, such as XL35 [12], which subsequently led to the naming of the family as "intelectins" (intestinal lectins) following the detection of a closely-related gene in mouse small intestinal Paneth cells by Komiya et al. [5]. Due to the cellular localisation of expression of this gene, currently denoted $I t \ln 1$ or Itlna, a role in innate immunity was postulated.

Human and mouse intelectins were shown to bind lactoferrin $[3,13]$, leading to the alternative nomenclature of "intestinal lactoferrin receptor" ( $L f r)$. Additionally, transcripts identical to human intelectin-1 (Itln1) were detected in omental fat tissue, and termed omentin by Yang et al. [14]. These authors and others [15] have investigated the metabolic significance of Itln1, which is present in blood plasma and is thought to function as an adipocytokine. Circulating levels are inversely correlated to body mass index [15] and to occurrence of type-1 diabetes mellitus [16]. A single nucleotide polymorphism in It $\ln 1$ has also been implicated in susceptibility to Crohn's disease [17], and a closely-linked 
coding SNP was also found to be associated with incidence of asthma [18].

Intelectin expression in the gut and lung mucosa is known to be highly up-regulated in the immune response to parasitic infections and Th2-cytokine dominated allergic responses [19-22]. Whilst Itln1 is expressed in Paneth cells at a high level in normal mouse small intestine, infection of $\mathrm{BALB} / \mathrm{c}$ mice with the small intestinal dwelling helminth Trichinella spiralis, induced de novo expression of an additional gene termed intelectin-2 (Itln2) in goblet cells, which was secreted into the mucus layer [6]. This gene, alternatively termed Itlnb, is absent from the reference C57BL/6J genome, and it was suggested that lack of It $\ln 2 / I t \ln b$ may be partially responsible for the delayed expulsion of T. spiralis in C57BL/6J mice compared to BALB/c [6].

In a subsequent genome-wide screen for gene copy number variation (CNV), Graubert et al. [23] highlighted the Itln locus on distal chromosome 1 as a hotspot of gene duplication in most mouse strains analysed. Gene duplication [24] represents an evolutionary mechanism whereby populations can adapt to increase the effective dosage of an advantageous gene in the face of a particular selection pressure, such as the burden of intestinal parasites. Once fixed, duplicated genes (paralogs) can evolve distinct functions. In the context of responses to parasites, a notable example of gene duplication in the mouse is at the mast cell proteinase locus on chromosome 14 [25]. Mast cell proteinases contribute anti-parasite effector mechanisms, and the gene Mcpt1 has been shown to enhance expulsion of T. spiralis [26].

In this study, we aimed to sequence the $I t \ln$ locus in a non-C57BL/6J strain, in order to characterise the nature and extent of duplication of mouse $I t l n$ genes. We focussed on the 129S7 mouse, as a BAC library was available for this strain [27], which is commonly used in gene deletion studies, and this information would enable the design of future specific Itln knockout strategies.

\section{Results}

\section{Sequencing of 12957 It In locus}

The Itln locus on chromosome 1 of the Celera mouse assembly [28] shows multiple copies of Itln genes as compared to that of the C57BL/6J reference genome. However, it is an assembly consisting of a mix of 5 different mouse strains (129X1/SvJ, 129S1/SvImJ, DBA/2J, $\mathrm{A} / \mathrm{J}$ and $\mathrm{C} 57 \mathrm{BL} / 6 \mathrm{~J})$ and there are about $43 \mathrm{kbp}$ of gaps on this locus. To improve on the annotation and resolve the exact structure of the 129S7 Itln copy number variations, 4 flanking bacterial artificial chromosome (BAC) clones were sequenced to a depth of $\sim 4.7 \times$ using the conventional Sanger sequencing method while the remaining middle two were sequenced to a much greater depth of $\sim 300 \times$ using the Illumina paired-end sequencing method. In view of the expected repetitive nature of the Itln locus, the use of this tiling path sequencing strategy was necessary to minimise errors in the assembly. Nevertheless, several gaps estimated (based on the size of the individual BAC clones) to be less than $10 \mathrm{kbp}$ in total are yet to be bridged. Gap closure over repeat elements with high level of sequence identity remains computationally challenging. The final assembly of the 6 BAC clones yielded a scaffold of about $603 \mathrm{kbp}$ (Figure 1); adding approximately $16 \mathrm{kbp}$ of sequences to the corresponding Celera assembly. The overall sequence identity between the two is about $97 \%$. However, there exist variable regions with sequence identity as low as $60 \%$ (Additional file 1) suggesting potential errors in the assembly using mixed strains of mouse. The sequence and annotations of the scaffold have been deposited in the GenBank with the accession number HM370554 and a graphical representation is also available at http://www.ncbi.nlm.nih.gov/nuccore/ 300085578?report=graph.

\section{Analysis of the 12957 ItIn locus}

A dotplot analysis (Figure 2) was first used to pinpoint regions of high similarity where the Itln genes may be located. Six complete or partial $\sim 62 \mathrm{kbp}$ tandemly or invertedly duplicated segments could be identified on the locus. Each of these segments is found to be flanked by murine endogenous retroviral (ERV) elements which have been known to mediate the host recombination [29]. These ERV elements may well serve as the breakpoint for

Pseudogenes
BAC clones
Figure 1 Schematic representation of the 129S7 It/n locus. Genomic DNA from six overlapping 12957 BAC clones was sequenced and
assembled as described in Materials and Methods to give a r 600 kbp final assembly. The locations of genes and pseudogenes are indicated.




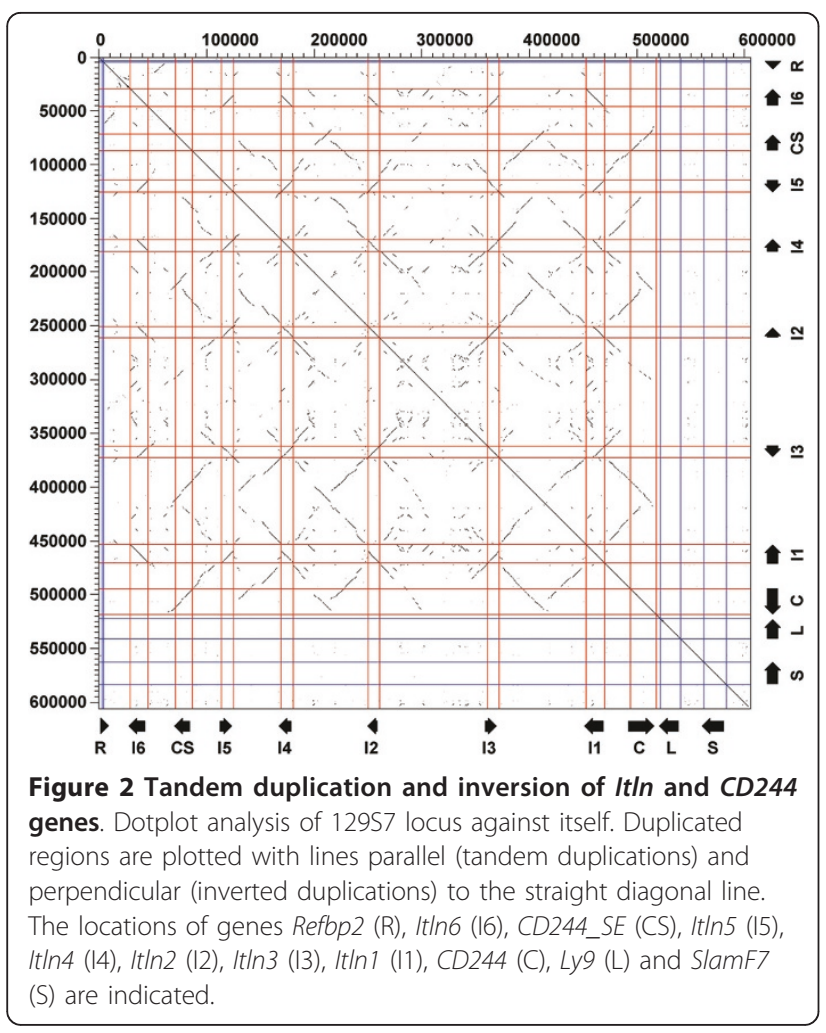

the recombination and expansion of the Itln locus. It is perhaps no coincidence that about $43 \%$ of the $129 \mathrm{~S} 7 \mathrm{Itln}$ locus is made up of repeat elements. This is significantly denser than the average $37.5 \%$ observed for the whole genome in the C57BL/6J mouse [30].

In addition to the flanking genes (Refbp $2, L y 9$ and Slamf7), the $\sim 500 \mathrm{kbp}$ Itln locus has been predicted to be made up of 5 full-length $I t \ln$ variants (Itln1-2,4-6), one truncated It $\ln (I t \ln 3), 6$ pseudo-Itln, one CD244 variant CD244_SE, one full-length CD244 and 5 pseudoCD244 (Figure 1 \& Additional file 2). The 6 Itln genes can be further classified into two categories based on their sizes of either $\sim 11$ or $\sim 17 \mathrm{kbp}$; with $I t \ln 1$ and $I t \ln 6$ having additional LINE/L1 retrotransposable elements inserted into their respective intron 7. In fact, variations also exist in the organisation of the pseduogenes on each of the duplicated segments; with some having different exons removed.

To further investigate the evolutionary basis of the seemingly active segmental duplication, an unrooted neighbour-joining tree (Figure 3) was constructed using intron 5 and its equivalent in all the Itln genes and pseudogenes, respectively. With less selection pressure than the exons, introns are known to mutate more rapidly and uniformly; making them an informative phylogenomic marker. The tree shows that the full-length genes and pseudogenes are clearly separated into two distinct clades. The clustering of the pseudogenes

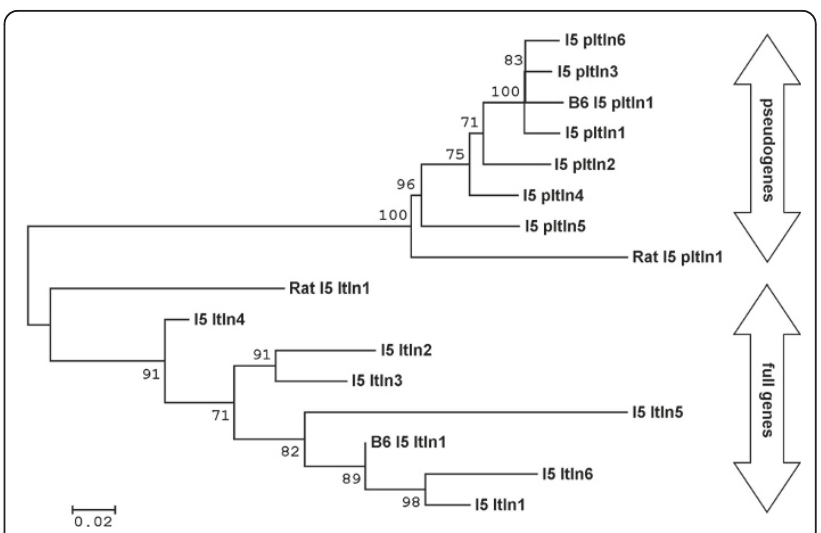

Figure 3 Phylogenetic analysis of the $I t / n$ genes and pseudogenes in 129S7, C57BL/6J and rat. The unrooted phylogenetic tree was constructed based on the sequences of Intron 5 (15) which range from about $400 \mathrm{bp}$ to $3.6 \mathrm{kbp}$. Numbers at the beginning of nodes represent the percentage bootstrap support. The full-length (It|n)- and pseudo-genes (plt|n) separate into two distinct clades. All C57BL/6J-derived (pseudo)genes are prefixed "B6".

suggests that they probably propagate by serial duplication of an existing pseudogene which may already be present within the duplicated segments together with its full-length gene. Detailed sequence analysis of each of the introns shows that the insertions of ERV and/or LINE repeats probably account for the branching of each genes and pseudogenes.

Although all 8 exons of the Itln genes can be mapped onto the 6 variants and their predicted mRNA sequences are highly conserved (94 to $97 \%$ sequence identity), they are predicted to be expressed and probably regulated differently. Both $I t \ln 3$ and $I t \ln 4$ transcripts are found to contain an early stop codon. While this is most likely to result in a truncated Itln3, the presence of potential ribosomal -1 frameshift sites identified further upstream of the stop codon at positions 175 and 177 is predicted to allow the translation of the fulllength Itln4 protein to proceed. Sequence conservation among the 5 predicted full-length Itln proteins ranges from 91 to $96 \%$. Not surprisingly, similar conservation is also observed when comparing the variants with the Itln1 [GenBank:NP_034714] of C57BL/6); with the Itln1 from both strains being identical to each other on the protein level. However, higher degrees of variation are found among the 5 Itln variants and the predicted Celera homologues; with the protein sequence identity ranges from 71 to $100 \%$ (Additional file 1). Two of Celera's Itln variants may also contain sites for ribosomal -1 frameshifting. The Itln 1 and $I t \ln 2$ genes of $129 \mathrm{~S} 7$ are found to be identical to Celera's Itlna [GenBank: NP_034714] and Itlnb [GenBank:NP_001007553] respectively. The observed variation in the remaining $I t \ln$ 
variants is probably the result of a mixed Itln population derived from the 5 mouse strains.

CD244_SE was initially predicted to be a CD244 pseudogene due the lack of the terminal exon 9. However, a closer inspection has resulted in the identification of a potential internal splicing site in the predicted exon 3. Comparison of all the probable coding sequences derived from this internal splicing site has led to the prediction of a protein which shares $65 \%$ identity with the soluble form of rat CD244 [31]. This novel protein is made up of 4 exons which are highly similar ( $90 \%$ conservation) to that of CD244; with part of exon 3 spliced to exon 5 . On the other hand, it is also interesting to note that the CD244 protein sequence of $129 \mathrm{~S} 7$ and Celera [GenBank:XP_001003781] are almost identical with $99 \%$ sequence identity but they share only $89 \%$ sequence identity with that of C57BL/6J [GenBank: NP_061199].

All the pseudogenes contain exons that share at least $70 \%$ sequence identity with their full-length counterparts. None of them contains the full set of exons and multiple stop codons are often spread across them.

\section{Southern blot analysis}

Southern blot analysis (Figure 4) was conducted to experimentally verify both the assembly and the dotplot analysis on the number of duplicated segments. Using probes (Additional file 3) that hybridised to the 5' and 3' end of the Itln genes, fragments gave a banding pattern that was consistent with that predicted from the in silico restriction analysis of the locus. In addition to the 129S7 strain, Southern blotting was also carried out on C57BL/ 6J, 129X1, 129S1, DBA2/J and A/J mice (Figure 4). All the 129 substrains also showed a banding pattern that was consistent with restriction fragments predicted from the sequenced 129S7 Itln locus. DBA2/J and A/J strains showed a similar pattern, but with the appearance of a prominent extra band with the 3' probe. Although it is highly likely that these four mouse strains share a similar genomic organisation at the Itln locus with the 129S7 substrains, differences observed in the Southern analysis should further warrant a note of care when using the mixed assembly of Celera's mouse genome. The C57BL/6J strain consistently gave a single band, as expected.

\section{It In CNVs in mouse}

To date, 5 genome-wide CNV studies of varying degrees of resolution have been carried out in different mouse strains [23,32-35]. However, none has specified the exact copy number of the Itln genes. Recently, the Wellcome Trust Sanger Institute has sequenced 17 different mouse strains using the Illumina next generation sequencing technology. With the knowledge that there are 6 Itln genes in the $129 \mathrm{~S} 7$ mouse and that it has a similar Southern pattern to the other 129 substrains, an attempt to identify the Itln CNVs in these strains was done by comparing the sequencing coverage across the

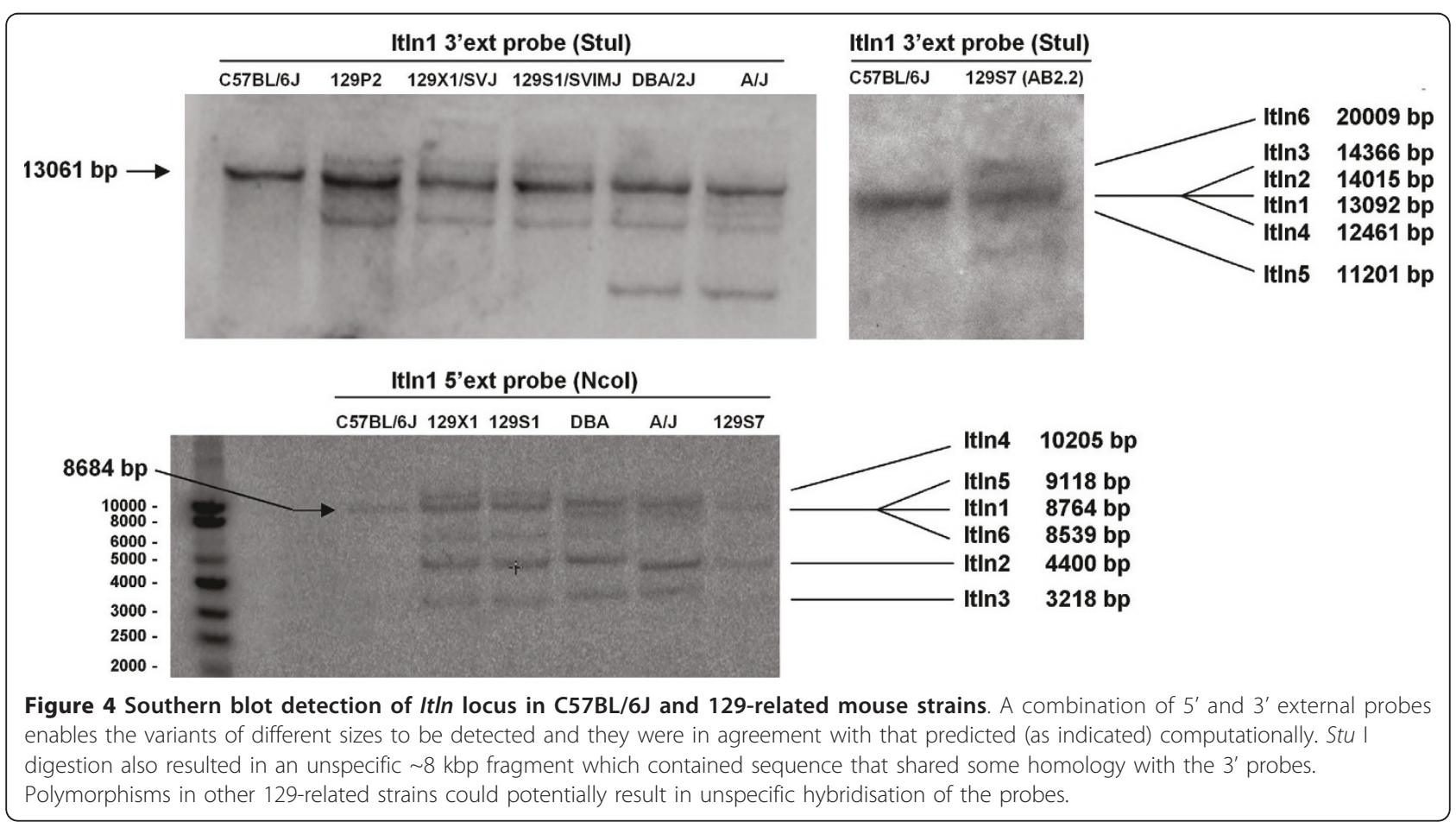


Itln locus (Figure $5 \&$ Additional file 4). During the mapping of these reads against the $\mathrm{C} 57 \mathrm{BL} / 6 \mathrm{~J}$ reference genome, reads are expected to collapse together to increase the mapping coverage at segments where copy number gains are located. This gain can be quantified by the $\log _{2}$ ratio of the coverage at a particular position; with for example a single (heterozygous) copy gain represented as $\log _{2}(3 / 2)=0.58$. A locus duplicated once in a homozygous inbred strain would be represented as $\log _{2}(4 / 2)=1$. Such a comparison is in fact analogous to $\mathrm{CNV}$ detection using the array comparative genomic hybridisation (aCGH) method [36].

Relative to the single Itln locus in the C57BL/6J reference mouse, an approximately $62 \mathrm{kbp}$ segment containing the Itln gene has apparently been duplicated several times in all the 16 strains investigated. Although the $\log _{2}$ ratio of the $\mathrm{CAST} / \mathrm{EiJ}$ strain falls close to the threshold (Additional file 4, Figure S3a), the consistently higher than reference coverage across the Itln locus (Figure 5) suggests that the segment may actually be duplicated once. The $\log _{2}$ ratio for the $129 \mathrm{~S} 1 / \mathrm{SvImJ}$ strain vs C57BL/6J varies between 1.8 and 2.7 over the Itln locus (Additional file 4, Figure S3a), well above the threshold for calling gene amplication, and suggesting 4 to 6-fold gain of the locus compared to C57BL/6J. Similar gain is also observed in the other strains (result not shown).

\section{Evidence for expression of mouse It ln variants}

Polymerase chain reaction analysis using common primers which amplified the Itln cDNA from all mouse tissues sampled, gave a product of the expected size (301 bp). Restriction enzyme analysis (Additional file 5) revealed variation in patterns across the different tissue samples, indicating heterogeneity of Itln variant expression. PCR products were sequenced to confirm the identities of variants expressed in different tissues (summarised in Table 1). Briefly, Itln2 was expressed in trachea and $\operatorname{Itln} 1$ was the dominant form expressed in small intestinal tissues, whereas $I t \ln 6$ was dominant in stomach and caecum. Since there was evidence for expression of both $I t \ln 2$ and $I t \ln 6$ in colon, the PCR product was cloned and 48 clone sequences were obtained. Twenty six clones were consistent (>98\% identity) with $I t \ln 2$ and 12 were consistent with $I t \ln 6$. The remaining 10 sequences contained ambiguities and/or were not consistent with any of the six known Itln variants.

\section{Detection of putative Nkx3.1 transcription factor binding} sites in ItIn promoter regions

In C57BL/6J, the so-called "Int5" promoter region of Itln1, which we have renamed Promoter Region 5 (Pr5) to avoid confusion, has been found to harbour the binding site for the transcription factor $\mathrm{Nkx3.1}$ [37] at

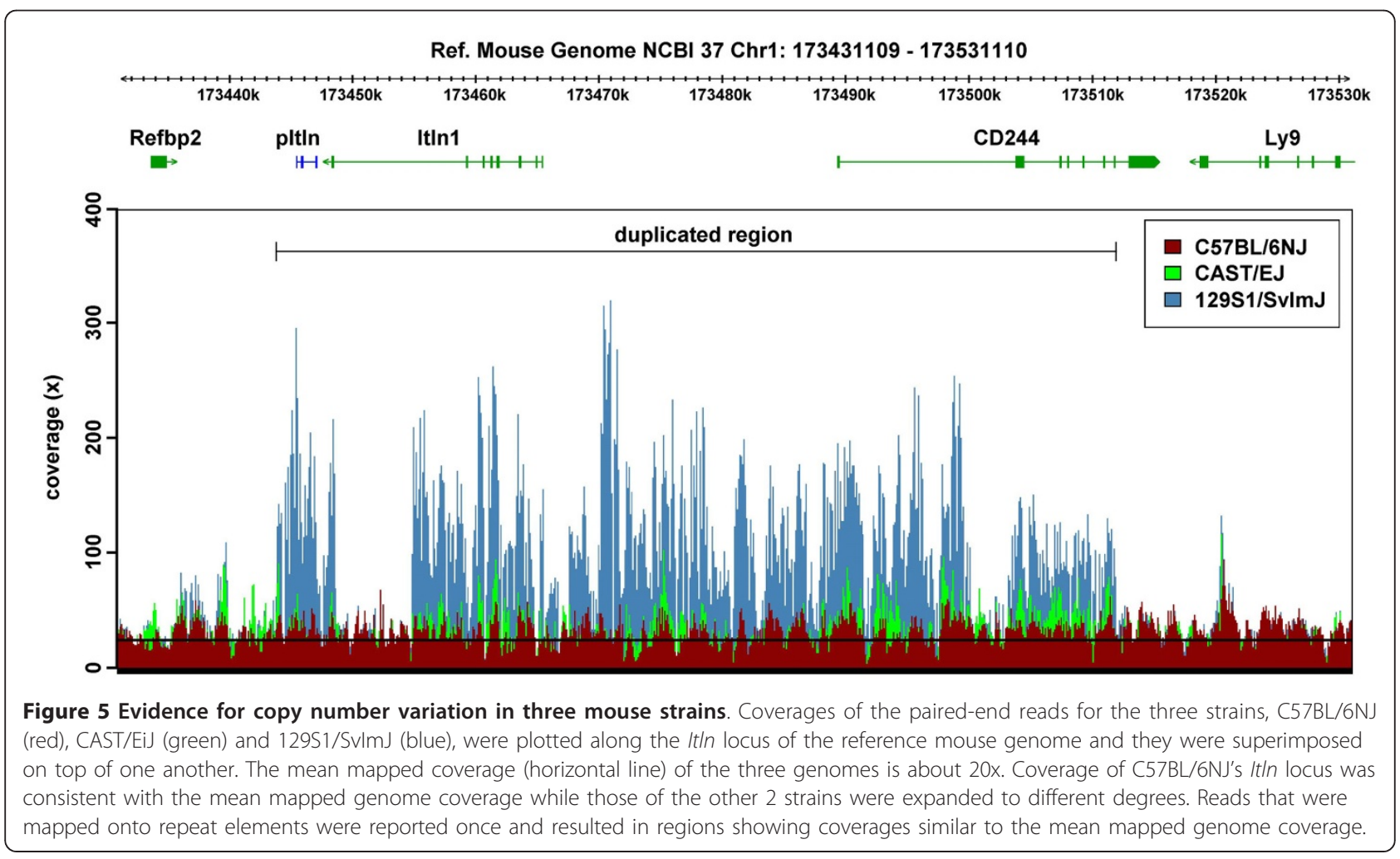


Table 1 It In variants identified in various mouse tissues

\begin{tabular}{|c|c|}
\hline Tissue & ItIn variant identified \\
\hline Trachea & $1 t \ln 2$ \\
\hline Stomach & $1 t \ln 6$ \\
\hline Duodenum & $\mid t \ln 1$ \\
\hline Jejunum & $\mid t \ln 1$ \\
\hline Ileum & $\mid t \ln 1$ \\
\hline Caecum & $1 \operatorname{tgn} 6$ \\
\hline Colon & $1 t \ln 2 \& \mid t \ln 6$ \\
\hline
\end{tabular}

Summary of the RT-PCR and sequencing of pan-ItIn PCR products show tissue specific expression of the variants. Similar results were obtained with $129 \mathrm{~S} 2$ and 129P2 tissues (Additional file 5). These results do not preclude the presence of additional It In variants in any tissue.

position -1348 to -1343 . The cognate DNA binding sequence for $\mathrm{Nkx3} .1$ was determined by Steadman et al. [38] to be TAAGT(A/G). This Pr5 promoter region is found to be conserved in $I t \ln 1, I t \ln 2, I t \ln 3$ and $I t \ln 6$ of the 129S7 mouse (Figure 6). Since Itln3 is predicted to be a truncated protein, it was excluded in the subsequent search for the Nkx3.1 binding site. Putative binding sites on the promoter of $I t \ln 1, I t \ln 2$ and $I t \ln 6$ are predicted to be located at positions -1357 to $-1351,-1379$ to -1373 , and -3747 to -3741 respectively (Table 2 ).

However, the "TAAGTG" motif is only conserved between the $I t \ln 1$ promoter of C57BL/6J and $129 \mathrm{~S} 7$. A single bp variation results in a "TAAATG" binding motif on the promoters of Itln 2 and $I t \ln 6$ which may in turn affect the binding affinity for Nkx3.1.

\section{Discussion}

We present here the sequence of the Itln locus on mouse chromosome 1 in a non-C57BL/6J mouse strain (129S7). Multiple duplications of Itln and CD244 genes/ pseudogenes have resulted in the expansion of the locus in comparison to that found in the reference $\mathrm{C} 57 \mathrm{BL} / 6 \mathrm{~J}$

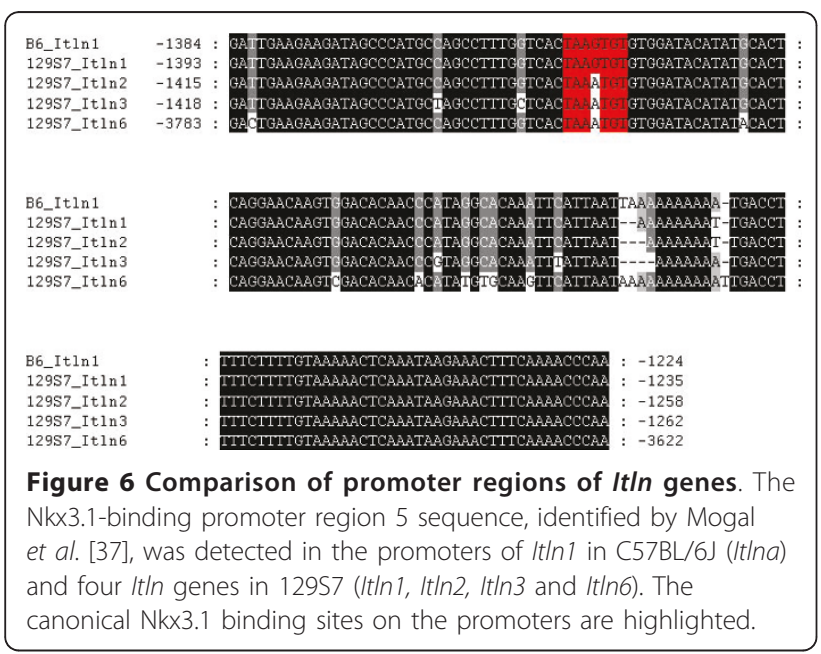

Table 2 In silico prediction of Nkx3.1 binding sites in 12957 ItIn promoters

\begin{tabular}{lllllll}
\hline It $\ln$ & Model & Score & Relative score & Start & End & site sequence \\
\hline It $\ln 1$ & NKX3-1 & 8.333 & 0.883282576 & -1357 & -1351 & TAAGTGT \\
\hline It $\ln 2$ & NKX3-1 & 5.085 & 0.766546505 & -1379 & -1373 & TAAATGT \\
\hline It $\ln 6$ & NKX3-1 & 5.085 & 0.766546505 & -3747 & -3741 & TAAATGT \\
\hline JASPAR scan for & & &
\end{tabular}

JASPAR scan for the conserved Nkx3.1 motif in the 5 kbp upstream (represented by the negative positions) promoter regions.

mouse. Nearly half of this expanded Itln locus comprises repeat sequence elements. It is likely that the presence of flanking ERV and LINE elements was involved in the duplications and inversion of the Itln locus; and that the events may be relatively recent and active [29,39].

The present data allows us to unequivocally define the genetic architecture of the Itln locus in the 129S7 genome. It bears considerable similarity to that obtained in the Celera mouse genome but with reduced homology over the coding regions. This is most likely due to the Celera sequence being a composite of 5 different strains and the difficulty in resolving repetitive sequences; and hence not necessarily representative of any one strain. It has been estimated that as much as $57 \%$ of highly identical segmental duplications in the mouse genome were potentially misassembled and that segmental duplications/CNVs make up 1.7-2.0\% of the mouse genome [40]. We have overcome this pitfall by a combined strategy of tiling path sequencing of BAC clones and Southern verification.

Furthermore, our Southern blot data suggested that other non-C57BL/6J strains also have a similar structure. In support of this, not only have a number of previous genome-wide studies on the structural variations of the mouse genome identified this locus as a hotspot of recombination and CNV, the ENSEMBL annotated Itln protein family ENSFM00250000003313 also reports variations in the copy number of the Itln gene in different animal species. The majority of the 17 mouse strains show evidence of expansion at this locus too, raising the question as to whether the Itln locus has been deleted in the $\mathrm{C} 57 \mathrm{BL} / 6 \mathrm{~J}$ and related strains, or alternatively expanded in a common ancestor to the 129S7 and other non-C57BL/6J strains.

It was not known whether CNV at the mouse Itln locus confers a simple dosage effect, or alternatively, whether each variant plays a different functional role. We found that in contrast to the C57BL/6J mouse strain, which expresses a single $I t \ln 1$ gene [30], 129S2 and 129P2 mice exhibited site-specific expression of at least three different Itln variants along the gastrointestinal tract. Such differential tissue expression of Itln variants has also been observed in the channel catfish [41]. This highly selective expression pattern and the minor 
amino acid sequence differences between the Itln variants suggest that these duplicated genes may be of functional significance at the different sites of expression. It has been suggested that intelectins play a role in modifying mucus properties, and therefore site-selective expression of variants may be important in the same way that mucosal trefoil factor variant expression tends to be co-ordinated with specific mucin types in different regions of the gastrointestinal tract [42]. Similarly, sheep intelectin variants have been found to exhibit site-specific expression differences between airways, gastric stomach (abomasum) and small intestine [7]. However, the consequences to C57BL/6J mice, if any, of lack of expression of Itln 2 and Itln 6 in the GI tract, remain to be established.

It has been shown that a functional Nkx3.1 transcription factor binding site is located in the mouse $I t \ln 1$ promoter [37]. Nkx3.1 is highly expressed in the prostate, where it is believed to function as a tumour repressor gene [43], and regulates Itln1 expression in prostate epithelial cells [44]. Haploinsufficiency in $N k x 3.1$, where only a single functional allele of the gene is present, was found to result in reduced expression of a range of dosage-sensitive genes in the mouse prostate, including $I t \ln 1$, expression of which was essentially lost in $N k x 3.1^{+/-}$mice [44]. Importantly, Itln1 expression was itself shown to suppress prostate cell growth [37], and thus Itln1 appears to be an effector of prostate cancer repression. It was of interest therefore to determine the occurrence of Nkx3.1 binding sites in all six Itln genes described here. The Pr 5 region which was found to contain the Nkx3.1 binding sequence TAAGTG [38] was present in the promoter regions of four of the six Itln genes but the binding region was mutated to TAAATG in all but $I t \ln 1$, suggesting that although $I t \ln 1$ probably remains under the transcriptional control of Nkx3.1 in 129S7 mice, the same is not necessarily true for the other Itln variants.

Importantly, an adipocytokine [14] role has been ascribed to human Itln1 (omentin-1), which can be detected in blood plasma and serum. Estimates for normal human serum Itln1 concentration vary from $20 \mathrm{ng} /$ $\mathrm{ml}$ [45] to over $100 \mathrm{ng} / \mathrm{ml}$ [14]. Recombinant Itln1 has been shown to stimulate insulin-dependent glucose uptake by adipocytes in vitro [14], and its expression in visceral fat and in the circulation is negatively correlated with body mass index and waist circumference [46] Weight loss and aerobic exercise were associated with significant increases in serum Itln1 levels [47] and a corresponding reduction in cardiometabolic risk factors.

With reference to the comparative metabolic significance of Itln expression in mice, Orozco et al. [48] investigated the influence of $\mathrm{CNV}$ on metabolic traits using a combined genomic - metabolomic approach in the $\mathrm{C} 57 \mathrm{BL} / 6 \mathrm{~J}(\mathrm{~B} 6)$ and $\mathrm{C} 3 \mathrm{H} / \mathrm{HeJ}(\mathrm{C} 3 \mathrm{H})$ strains, and in $\mathrm{B} 6 \mathrm{xC} 3$ $\mathrm{H}$ crosses. Hotspots of copy number variation in chromosomes 1, 4 and 17 were associated with metabolic traits, and specifically, CNV in Itln1 (Itlna) was linked with weight, triglycerides, adiposity, glucose and insulin level [48]. Itln mRNA expression level, as detected by Agilent microarray analysis [48], was significantly elevated in the high copy number genotype $(\mathrm{C} 3 \mathrm{H})$, when assayed in adipose, brain and liver tissue. The detailed characterisation of the Itln locus described here provides new specific candidates for further investigation of the role of $I t l n$ genes in mouse models of metabolic disorders.

In addition to $I t \ln$, the $C D 244$ gene sequence is also amplified within the 129S7 locus. Specifically, this study has identified a putative CD244 variant, analogous to the secreted CD244 variant previously described in the rat [31]. However, we found no evidence of expression of the corresponding transcript by RT-PCR in mouse small intestine (data not shown), so it is not clear whether it is actively transcribed in this mouse strain. CD244 (also known as 2B4) is a member of the SLAM family of cell surface receptors, which are present as a gene cluster adjacent to the Itln locus on mouse and human chromosome 1. A range of lymphocytes, including natural killer (NK) cells and $\mathrm{CD}^{+} \mathrm{T}$-cell subsets have been shown to express CD244, which is the high affinity counter-receptor for CD48 (for a review, see [49]). While engagement of human NK cell CD244 with CD48 results in enhanced killing of CD48-expressing cells [50], the situation appears more complex in mice, where both activating and inhibitory roles have been demonstrated [51]. It is known that $\mathrm{C} 57 \mathrm{BL} / 6 \mathrm{~J}$ mice possess a single $C D 244$ gene, expressed as long and short splice variants, which are distinct from CD244 cDNAs amplified from other mouse strains [52]. The potential expression of a soluble CD244 variant suggested by this study, may introduce an additional level of regulation of lymphocyte interactions in non- C57BL/6J -related strains, and may therefore contribute to immunological differences between C57BL/6J and other strains.

\section{Conclusions}

To conclude, we have determined the sequence for essentially the complete Itln locus in the $129 \mathrm{~S} 7$ mouse, as a prototype for non-C57BL/6J mouse strains. This has elucidated the nature of the copy number variation occurring at this locus, arising from tandem duplication of Itln and CD244 genes. Individual Itln genes showed strong tissue expression specificity while most duplicated CD244 genes were non-functional.

\section{Methods}

\section{Identification and sequencing of It/n-containing BAC clones}

The Itln locus of the $129 / \mathrm{Sv}$ mouse was characterised by sequencing BAC clones derived from the AB2.2 ES cell 
of the $129 \mathrm{~S} 7 / \mathrm{SvEvBrd}-\mathrm{Hprt}^{\mathrm{b}-\mathrm{m} 2}$ substrain [27]. Since the reference $\mathrm{C} 57 \mathrm{BL} / 6 \mathrm{~J}$ mouse genome contains only a single copy of the Itln gene (Additional file 2), two BAC clones flanking the gene were first identified from the BAC end sequences aligned to the ENSEMBL Mouse Assembly (ENSEMBL release 46, NCBI m36) [53]. A tiling path of candidate BAC clones moving towards each other from the two ends of the locus (Figure 1) was then built by selecting BAC clones with end sequences that matched to the sequenced ones. Two iterations of blast search were done to identify these matching clones; an initial identity cut-off of $90 \%$ was applied, followed by quality trimming of the filtered sequences and a final round of blast with $98 \%$ identity cut-off. PCRs to check the presence of Itln were also performed to further ensure the correct candidates were picked. A total of six overlapping BAC clones (bMQ411i17, bMQ453f04, bMQ285e14, bMQ_239m09, bMQ312m04 and bMQ302g15) spanning the entire Itln locus were purchased from Geneservice (UK). BAC clones were grown in LB broth containing chloramphenicol (12.5 $\mu \mathrm{g} / \mathrm{ml}$ ) and DNA was purified using a proprietary kit (NucleoBond ${ }^{\circledR}$ BAC 100, Macherey-Nagel). Four shortgun libraries with insert size of about $2 \mathrm{kbp}$ were constructed from the four respective flanking clones (bMQ411i17, bMQ453f04, bMQ312m04 and bMQ302g15) and sequenced by GATC Biotech (Konstanz, Germany) using the ABI Big Dye Terminator Mix v3.0 in a ABI 3730 sequencing machine. Gap closures for these four clones were achieved by primer walking. 36 bp paired-end next-generation sequencing using the Illumina Genome Analyzer II was carried out by ARKGenomics (Roslin, UK) on the two remaining middle clones (bMQ_239m09 and bMQ312m04).

\section{Sequence assembly}

Sequences from each of the four shortgun libraries were assembled using Phred (v 0.020425.c)/Phrap (v1.080812)/Consed (v15.0) [54] into their respective scaffolds. Parameters for the assembly were set to high stringency: vector scanning, 32bp trimming of all 5' ends, minimum Phred score of 20, minimum length of matching word increased to 30, and level of contigs merging stringency set to the highest. Contaminating contigs from $E$. coli were removed by blasting them against the NCBI's E. coli genomes [55]. All assembled contigs were also manually inspected to correct for errors due to duplicated segments and repeats; with special attention paid to regions having higher than $97 \%$ cross_match (v 1.080812) [54] identity. They were ordered into a single scaffold for each of the libraries with the aid of the forward/reverse pairing data and locations of the predicted coding sequences.
A strategy involving three iterations of mapping against reference sequences, de novo sequence assembly and a final scaffold construction was employed to assemble the remaining two BAC clones. The 4.8 million $36 \mathrm{bp}$ Illumina paired-end reads were adaptor- and quality-trimmed to $32 \mathrm{bp}$ with the fastx toolkit (v0.0.11) [56] to ensure only reads with phred scores higher than 15 were kept. These processed reads were first mapped against the contaminating $E$. coli str. K-12 substr. DH1OB [GenBank:NC_010473] reference genome and pBACe3.6 [GenBank:U80929] cloning vector using MAQ (v0.7.1) [57]. The 2.4 million clean unmapped reads next underwent a second round of mapping, again with MAQ, to remove sequences overlapping with the known flanking upstream BAC clones. Reads which were unmapped, containing indels or more than one mismatch and mapping quality score less than 20 amounted to about 1.2 million. They were finally MAQ mapped, allowing 2 mismatches, against the corresponding Itln locus of Celera's mouse assembly. Again the unmapped reads were extracted and input into the de novo assembly program, Velvet (v0.7.54) [58] to obtain a set of contigs that should cover the gaps in the MAQ mapped consensus sequence. In addition, de novo assembly using Velvet on the 2.4 million reads extracted from the first mapping exercise was also done to facilitate the resolution of errors in the MAQ consensus sequence. The Velvet optimiser script was used and no scaffolding was done. Contigs with exceptionally high or low k-mer coverage values were also discarded. The scaffold for these two BAC clones was constructed by merging together the final MAQ consensus sequence and the two sets of Velvet contigs using the SeqMan module of Lasergene (v8.1). Merging was only allowed where end sequences overlapped for at least $15 \mathrm{bp}$ with 99\% identity. Where necessary manual correction was always performed. The final single scaffold of the six BAC clones was also assembled with the SeqMan.

\section{Annotation}

Spidey (v1.40) [59] and Blast2seq (v2.2.20) [60] were used initially to search and predict the gene organisation of the 6 known genes, namely Refbp2, It $\ln 1, \operatorname{It} \ln 2, C D 244$, Ly9 and Slamf7, on the new 129S7 Itln locus. The reference mRNA sequences and the intron/exon boundaries used were obtained from both NCBI Refseq and ENSEMBL gene transcripts (Additional file 2). Splicing junctions were manually corrected to reflect the exact acceptor/donor sites. Pseudogenes were only annotated when their exons share at least $70 \%$ identity to the coding sequences. In addition, the exon-exon junctions of the predicted transcripts were predicted with the RNASPL program of the Softberry web server [61] to check for alternative splicing. Potential frame-shifting in the 
transcripts were checked with the web tool KnotInFrame [62]. Repeat elements on the locus were identified using the program RepeatMasker (v3.2.7) [63].

\section{Comparative genomics}

Dotplot and blastz analyses between the Itln locus of the 129S7 strain and itself and that of the Celera mouse assembly were carried out using dotter (v3.1) [64] and zPicture [65] to gain insights into the structural organisation of the locus. Intelectins of other animals were obtained from the ENSEMBL protein family ENSFM00250000003313. ClustalW (v1.83) [66] was used to align the mRNA and the translated protein sequences of the different intelectins while MUSCLE [67] was the program of choice for the multiple sequence alignment of the genomic and the intronic sequences. For the phylogenetic analysis, the alignment of intron 5 sequences of the full length and pseudo-Itln genes from 129S7, C57BL/6J and rat was manually adjusted before being analysed with the MEGA (v5.0 beta) software suite [68]. The tree was constructed using the neighbour-joining methodology with the branch distances computed based on the Kimura-2-parameter model. All ambiguous positions were removed for each sequence pair. The final consensus tree was inferred from 1000 bootstrap replicates. The presence of the Itln CNV in 17 other mouse strains (129P2/OlaHsd, 129S1/SvImJ, 129S5/SvEvBrd, A/ J, AKR/J, BALB/cJ, C3H/HeJ, C57BL/6NJ, CAST/EiJ, CBA/J, DBA/2J, LP/J, NOD/ShiLtJ, NZO/HiLtJ, PWK/ $\mathrm{PhJ}$, Spret/EiJ and WSB/EiJ) was detected by using CNVseq [36] with the threshold of the $\log _{2}$ ratio set to 0.6. Furthermore, the relatively high sequencing coverage, averaging 20.1 fold, of the 17 genomes allows the use of a small sliding window size of about $4 \mathrm{~kb}$ and a $p$-value of $10^{-5}$ to increase the CNV resolution (results not shown). The zoom-in coverage plots of the identified Itln CNV were drawn by the plotrix library of R [69]. Sequencing data of these mice were obtained from the Wellcome Trust Sanger Institute ftp://ftp.sanger.ac.uk/pub/mouse_genomes/.

\section{Nkx3.1 transcription factor binding sites}

The potential binding sites for the Nkx3.1 transcription factor on the $5 \mathrm{kbp}$ (upstream of the transcription start site) promoters of the six 129S7 Itln variants and that of the C57BL/6J were predicted using three different web tools, namely JASPAR scan of individual promoters applying $75 \%$ relative profile score threshold [70]; zPicture pairwise alignment between C57BL/6J and 129S7 promoters followed by rVista [71] search for conserved TAA[G/A]T[A/G][A/C/T] binding sites; and MEME motif discovery [72]. Results from the three predictions were next compared to identify conserved binding sites that fall on the evolutionary conserved regions conserved between C57BL/6J and 129S7. Where no such region exists between C57BL/6J and 129S7, sites with the highest similarity to the C57BL/6J's TAAGTG motif were picked.

\section{Southern blotting}

Two probes (Additional file 3) were designed to sit on the 5' and 3' end of the Itln genes. The probe sequences were selected based on the conserved regions of the $6 \mathrm{Itln}$ variants. There were blasted against the reference mouse genome to make sure that they do not hybridise to other parts of the genome. Southern blots were done according to the standard protocols of Southern [73]. Briefly, genomic DNA from 7 mouse strains (C57BL/6J, 129P2, 129S1/SvImJ, A/J, DBA/2J, 129X1/SvJ and 129S7) were digested over night at $37^{\circ} \mathrm{C}$ with either Nco I or Stu I for hybridising with either the $5^{\prime}$ or $3^{\prime}$ probe respectively. DNAs were then separated on $0.8 \%$ agarose gel overnight at $20 \mathrm{~V}$ before being transferred to the nylon membrane for hybridisation with the radioactive probes.

\section{Evidence for expression of Itln variants}

Samples of the following tissues were collected from healthy uninfected mice of the 129S2 and 129P2 strains: trachea, stomach, duodenum, jejunum, ileum, caecum and colon. The samples were collected into RNAlater and subsequently RNA was extracted, reverse transcribed and then amplified by PCR using common primers for all six Itln variants (ITLN_all_F: TCAGCTAGCAACTCT CAGCTCCT; ITLN_all_R ACACTAGCCACCAGGGT CCA; 35 cycles, annealing temp: $57^{\circ} \mathrm{C}$ ). PCR products were sequenced and results analysed for evidence of a predominant $I t \ln$ variant, or evidence of a mixture. Additionally, PCR products were digested with restriction enzymes Hha I, Mbo II and Hae III (see Additional file 5 for specificities). The PCR products from $129 \mathrm{~S} 2$ colon and $129 \mathrm{P} 2$ trachea were subjected to TOPO cloning. Positive clones from 129S2 colon (48) and 129P2 trachea (4) were individually sequenced.

\section{Additional material}

\footnotetext{
Additional file 1: Comparison of ItIn locus from 129S7 mice and the Celera mouse assembly. Alignment and dotplot showing the differences between the sequenced 12957 mouse and the corresponding Celera assembly.

Additional file 2: Genes in the It ln containing contigs of 129S7 C57BL/6J \& Celera mouse. A list of accession numbers for the coding sequences on the three assemblies.

Additional file 3: $5^{\prime}$ and $3^{\prime}$ probes used in the Southern blot Sequences of the Southern probes.

Additional file 4: Detection of ItIn CNV in non-C57/BL mouse strains using next-generation sequencing. Plots of $\log _{2}$ ratio and sequencing coverage at the It/n locus of different mouse strains.
} 
Additional file 5: Restriction enzyme analysis of It/n transcripts amplified from various tissues. Tissue specific expression of Itln variants in 12952 and $129 \mathrm{P} 2$ mouse.

\section{Acknowledgements}

We thank Dr Richard Talbot, ARK-Genomics, Roslin, UK, for performing Illumina sequencing and Dr Andrew Law, Roslin Institute, for helpful discussion on the sequence analysis. This work was funded by the Biotechnology and Biological Sciences Research Council (Award ref: BB/ E009069).

\section{Authors' contributions}

ZL performed the sequence assembly/analysis and co-wrote the manuscript. SW and AD performed the experimental procedures. AP and BW designed the study and AP co-wrote the manuscript. All authors have read and approved the final version

Received: 28 October 2010 Accepted: 16 February 2011 Published: 16 February 2011

\section{References}

1. Dehal P, Satou Y, Campbell RK, Chapman J, Degnan B, De Tomaso A, Davidson B, Di Gregorio A, Gelpke M, Goodstein DM, et al: The draft genome of Ciona intestinalis: insights into chordate and vertebrate origins. Science 2002, 298:2157-2167.

2. Tsuji S, Uehori J, Matsumoto M, Suzuki Y, Matsuhisa A, Toyoshima K, Seya T: Human intelectin is a novel soluble lectin that recognizes galactofuranose in carbohydrate chains of bacterial cell wall. Journal of Biological Chemistry 2001, 276:23456-23463.

3. Suzuki YA, Shin K, Lonnerdal B: Molecular cloning and functional expression of a human intestinal lactoferrin receptor. Biochemistry 2001, 40:15771-15779.

4. Lee JK, Schnee J, Pang M, Wolfert M, Baum LG, Moremen KW, Pierce M: Human homologs of the Xenopus oocyte cortical granule lectin XL35. Glycobiology 2001, 11:65-73

5. Komiya T, Tanigawa Y, Hirohashi S: Cloning of the novel gene intelectin which is expressed in intestinal paneth cells in mice. Biochemical and Biophysical Research Communications 1998, 251:759-762.

6. Pemberton A, Knight P, Gamble J, Colledge W, Lee J, Pierce M, Miller H: Innate BALB/c enteric epithelial responses to Trichinella spiralis: inducible expression of a novel goblet cell lectin, intelectin-2, and its natural deletion in C57BL/10 mice. Journal of Immunology 2004, 173:1894-1901.

7. French AT, Knight PA, Smith WD, Pate JA, Miller HR, Pemberton AD: Expression of three intelectins in sheep and response to a Th2 environment. Vet Res 2009, 40:53.

8. Blease SC, French AT, Knight PA, Gally DL, Pemberton AD: Bovine intelectins: CDNA sequencing and expression in the bovine intestine. Res Vet Sci 2009, 86:254-256.

9. Liao Y, Lopez V, Shafizadeh TB, Halsted CH, Lonnerdal B: Cloning of a pig homologue of the human lactoferrin receptor: Expression and localization during intestinal maturation in piglets. Comparative Biochemistry and Physiology Part A, Molecular \& Integrative Physiology 2007, 148:584-590.

10. Lindblad-Toh K, Wade CM, Mikkelsen TS, Karlsson EK, Jaffe DB, Kamal M, Clamp M, Chang JL, Kulbokas EJ, Zody MC, et al: Genome sequence, comparative analysis and haplotype structure of the domestic dog Nature 2005, 438:803-819.

11. Lee JK, Baum LG, Moremen K, Pierce M: The X-lectins: a new family with homology to the Xenopus laevis oocyte lectin XL-35. Glycoconj J 2004, 21:443-450.

12. Lee JK, Buckhaults P, Wilkes C, Teilhet M, King ML, Moremen KW, Pierce M: Cloning and expression of a Xenopus laevis oocyte lectin and characterization of its mRNA levels during early development. Glycobiology 1997, 7:367-372.

13. Suzuki YA, Lonnerdal B: Baculovirus expression of mouse lactoferrin receptor and tissue distribution in the mouse. Biometals 2004, 17:301-309.
14. Yang RZ, Lee MJ, Hu H, Pray J, Wu HB, Hansen BC, Shuldiner AR, Fried SK, McLenithan JC, Gong DW: Identification of omentin as a novel depotspecific adipokine in human adipose tissue: possible role in modulating insulin action. Am J Physiol Endocrinol Metab 2006, 290:E1253-1261.

15. Schaffler A, Neumeier M, Herfarth H, Furst A, Scholmerich J, Buchler C: Genomic structure of human omentin, a new adipocytokine expressed in omental adipose tissue. Biochim Biophys Acta 2005, 1732:96-102.

16. Tan BK, Pua S, Syed F, Lewandowski KC, O'Hare JP, Randeva HS: Decreased plasma omentin-1 levels in Type 1 diabetes mellitus. Diabet Med 2008, 25:1254-1255.

17. Barrett JC, Hansoul S, Nicolae DL, Cho JH, Duerr RH, Rioux JD, Brant SR, Silverberg MS, Taylor KD, Barmada MM, et al: Genome-wide association defines more than 30 distinct susceptibility loci for Crohn's disease. Nat Genet 2008, 40:955-962.

18. Pemberton AD, Rose-Zerilli MJ, Holloway JW, Gray RD, Holgate ST: A single nucleotide polymorphism in intelectin-1 is associated with increased asthma risk. Journal of Allergy and Clinical Immunology 2008, 122:1033-1034.

19. Pemberton AD, Knight PA, Wright SH, Miller HR: Proteomic analysis of mouse jejunal epithelium and its response to infection with the intestinal nematode, Trichinella spiralis. Proteomics 2004, 4:1101-1108.

20. Datta R, deSchoolmeester ML, Hedeler C, Paton N, Brass AM, Else KJ: Identification of novel genes in intestinal tissue which are regulated post infection with an intestinal nematode parasite. Infection and Immunity 2005, 73:4025-4033.

21. Kuperman DA, Lewis CC, Woodruff PG, Rodriguez MW, Yang YH, Dolganov GM, Fahy JV, Erle DJ: Dissecting asthma using focused transgenic modeling and functional genomics. J Allergy Clin Immunol 2005, 116:305-311.

22. Gu N, Kang G, Jin C, Xu Y, Zhang Z, Erle DJ, Zhen G: Intelectin is required for IL-13-induced monocyte chemotactic protein- 1 and -3 expression in lung epithelial cells and promotes allergic airway inflammation. Am J Physiol Lung Cell Mol Physiol 298:L290-296.

23. Graubert TA, Cahan P, Edwin D, Selzer RR, Richmond TA, Eis PS, Shannon WD, Li X, McLeod HL, Cheverud JM, Ley TJ: A High-Resolution Map of Segmental DNA Copy Number Variation in the Mouse Genome. PLoS Genet 2007, 3:e3.

24. Zhang J: Evolution by gene duplication: an update. Trends Ecol Evol 2003, 18:292-298.

25. Gimelbrant AA, Chess A: An epigenetic state associated with areas of gene duplication. Genome Res 2006, 16:723-729.

26. Knight PA, Wright SH, Lawrence CE, Paterson YY, Miller HR: Delayed expulsion of the nematode Trichinella spiralis in mice lacking the mucosal mast cell-specific granule chymase, mouse mast cell protease-1. Journal of Experimental Medicine 2000, 192:1849-1856.

27. Adams DJ, Quail MA, Cox T, van der Weyden L, Gorick BD, Su Q, Chan WI, Davies R, Bonfield JK, Law F, et al: A genome-wide, end-sequenced 129Sv BAC library resource for targeting vector construction. Genomics 2005, 86:753-758.

28. Mural RJ, Adams MD, Myers EW, Smith HO, Miklos GL, Wides R, Halpern A, Li PW, Sutton GG, Nadeau J, et al: A comparison of whole-genome shotgun-derived mouse chromosome 16 and the human genome. Science 2002, 296:1661-1671.

29. Jern P, Coffin JM: Effects of retroviruses on host genome function. Annu Rev Genet 2008, 42:709-732.

30. Waterston RH, Lindblad-Toh K, Birney E, Rogers J, Abril JF, Agarwal P, Agarwala R, Ainscough R, Alexandersson M, An P, et al: Initial sequencing and comparative analysis of the mouse genome. Nature 2002, 420:520-562.

31. Kumaresan PR, Stepp SE, Bennett M, Kumar V, Mathew PA: Molecular cloning of transmembrane and soluble forms of a novel rat natural killer cell receptor related to 2B4. Immunogenetics 2000, 51:306-313.

32. Cutler G, Marshall LA, Chin N, Baribault H, Kassner PD: Significant gene content variation characterizes the genomes of inbred mouse strains. Genome Res 2007, 17:1743-1754.

33. Egan CM, Sridhar S, Wigler M, Hall IM: Recurrent DNA copy number variation in the laboratory mouse. Nat Genet 2007, 39:1384-1389.

34. Cahan P, Li Y, Izumi M, Graubert TA: The impact of copy number variation on local gene expression in mouse hematopoietic stem and progenitor cells. Nat Genet 2009, 41:430-437. 
35. Quinlan AR, Clark RA, Sokolova S, Leibowitz ML, Zhang Y, Hurles ME, Mell JC, Hall IM: Genome-wide mapping and assembly of structural variant breakpoints in the mouse genome. Genome Res 2010, 20:623-635

36. Xie C, Tammi MT: CNV-seq, a new method to detect copy number variation using high-throughput sequencing. BMC Bioinformatics 2009, 10:80.

37. Mogal AP, van der Meer R, Crooke PS, Abdulkadir SA: Haploinsufficient prostate tumor suppression by Nkx3.1: a role for chromatin accessibility in dosage-sensitive gene regulation. J Biol Chem 2007, 282:25790-25800.

38. Steadman DJ, Giuffrida D, Gelmann EP: DNA-binding sequence of the human prostate-specific homeodomain protein NKX3.1. Nucleic Acids Res 2000, 28:2389-2395.

39. Belancio VP, Roy-Engel AM, Pochampally RR, Deininger P: Somatic expression of LINE-1 elements in human tissues. Nucleic Acids Res 2010, 38:3909-3922.

40. Bailey JA, Church DM, Ventura M, Rocchi M, Eichler EE: Analysis of segmental duplications and genome assembly in the mouse. Genome Res 2004, 14:789-801

41. Takano T, Sha Z, Peatman E, Terhune J, Liu H, Kucuktas H, Li P, Edholm ES, Wilson M, Liu Z: The two channel catfish intelectin genes exhibit highly differential patterns of tissue expression and regulation after infection with Edwardsiella ictaluri. Dev Comp Immunol 2008, 32:697-705.

42. Longman RJ, Douthwaite J, Sylvester PA, Poulsom R, Corfield AP, Thomas MG, Wright NA: Coordinated localisation of mucins and trefoil peptides in the ulcer associated cell lineage and the gastrointestinal mucosa. Gut 2000, 47:792-800.

43. Bhatia-Gaur R, Donjacour AA, Sciavolino PJ, Kim M, Desai N, Young P, Norton CR, Gridley T, Cardiff RD, Cunha GR, et al: Roles for Nkx3.1 in prostate development and cancer. Genes Dev 1999, 13:966-977.

44. Magee JA, Abdulkadir SA, Milbrandt J: Haploinsufficiency at the Nkx3.1 locus. A paradigm for stochastic, dosage-sensitive gene regulation during tumor initiation. Cancer Cell 2003, 3:273-283.

45. Pan HY, Guo L, Li Q: Changes of serum omentin-1 levels in normal subjects and in patients with impaired glucose regulation and with newly diagnosed and untreated type 2 diabetes. Diabetes Res Clin Pract 2010, 88:29-33.

46. Moreno-Navarrete JM, Catalan V, Ortega F, Gomez-Ambrosi J, Ricart W, Fruhbeck G, Fernandez-Real JM: Circulating omentin concentration increases after weight loss. Nutr Metab (Lond) 2010, 7:27.

47. Saremi A, Asghari M, Ghorbani A: Effects of aerobic training on serum omentin-1 and cardiometabolic risk factors in overweight and obese men. J Sports Sci 2010, 1-6.

48. Orozco LD, Cokus SJ, Ghazalpour A, Ingram-Drake L, Wang S, van Nas A, Che N, Araujo JA, Pellegrini M, Lusis AJ: Copy number variation influences gene expression and metabolic traits in mice. Hum Mol Genet 2009, 18:4118-4129.

49. Boles KS, Stepp SE, Bennett M, Kumar V, Mathew PA: 2B4 (CD244) and CS1: novel members of the CD2 subset of the immunoglobulin superfamily molecules expressed on natural killer cells and other leukocytes. Immunol Rev 2001, 181:234-249.

50. Nakajima $H$, Cella M, Langen $H$, Friedlein A, Colonna M: Activating interactions in human NK cell recognition: the role of 2B4-CD48. Eur J Immunol 1999, 29:1676-1683.

51. Chlewicki LK, Velikovsky CA, Balakrishnan V, Mariuzza RA, Kumar V: Molecular basis of the dual functions of 2B4 (CD244). J Immunol 2008, 180:8159-8167.

52. Kumaresan PR, Huynh VT, Mathew PA: Polymorphism in the $2 B 4$ gene of inbred mouse strains. Immunogenetics 2000, 51:758-761.

53. Ensembl Release 46. [http://aug2007.archive.ensembl.org/].

54. Gordon D, Abajian C, Green P: Consed: a graphical tool for sequence finishing. Genome Res 1998, 8:195-202.

55. NCBI microbial genomes BLAST. [http://www.ncbi.n/m.nih.gov/sutils/ genom_table.cgi].

56. FASTX-Toolkit. [http://hannonlab.cshl.edu/fastx_toolkit/].

57. Li H, Ruan J, Durbin R: Mapping short DNA sequencing reads and calling variants using mapping quality scores. Genome Res 2008, 18:1851-1858.

58. Zerbino DR, Birney E: Velvet: algorithms for de novo short read assembly using de Bruijn graphs. Genome Res 2008, 18:821-829.

59. Wheelan SJ, Church DM, Ostell JM: Spidey: a tool for mRNA-to-genomic alignments. Genome Res 2001, 11:1952-1957.
60. Altschul SF, Gish W, Miller W, Myers EW, Lipman DJ: Basic local alignment search tool. J Mol Biol 1990, 215:403-410.

61. Softberry - RNASPL. [http://linux1.softberry.com/berry.phtml? topic=rnaspl\&group=programs\&subgroup=gfind].

62. Theis C, Reeder J, Giegerich R: KnotInFrame: prediction of -1 ribosomal frameshift events. Nucleic Acids Res 2008, 36:6013-6020.

63. RepeatMasker Open 3.0. [http://www.repeatmasker.org]

64. Sonnhammer EL, Durbin R: A dot-matrix program with dynamic threshold control suited for genomic DNA and protein sequence analysis. Gene 1995, 167:GC1-10.

65. Ovcharenko I, Loots GG, Hardison RC, Miller W, Stubbs L: zPicture: dynamic alignment and visualization tool for analyzing conservation profiles. Genome Res 2004, 14:472-477.

66. Larkin MA, Blackshields G, Brown NP, Chenna R, McGettigan PA, McWilliam H, Valentin F, Wallace IM, Wilm A, Lopez R, et al: Clustal W and Clustal $\times$ version 2.0. Bioinformatics 2007, 23:2947-2948.

67. Edgar RC: MUSCLE: multiple sequence alignment with high accuracy and high throughput. Nucleic Acids Res 2004, 32:1792-1797.

68. Kumar S, Nei M, Dudley J, Tamura K: MEGA: a biologist-centric software for evolutionary analysis of DNA and protein sequences. Brief Bioinform 2008, 9:299-306, 69.

69. Lemon J: Plotrix, a package in the red light district of R. R-News 2006, 6:8-12.

70. Portales-Casamar E, Thongjuea S, Kwon AT, Arenillas D, Zhao X, Valen E, Yusuf D, Lenhard B, Wasserman WW, Sandelin A: JASPAR 2010: the greatly expanded open-access database of transcription factor binding profiles. Nucleic Acids Res 2010, 38:D105-110.

71. Loots GG, Ovcharenko I: rVISTA 2.0: evolutionary analysis of transcription factor binding sites. Nucleic Acids Res 2004, 32:W217-221

72. Bailey $T L$, Boden M, Whitington $T$, Machanick P: The value of positionspecific priors in motif discovery using MEME. BMC Bioinformatics 2010, 11:179.

73. Southern EM: Detection of specific sequences among DNA fragments separated by gel electrophoresis. J Mol Biol 1975, 98:503-517.

doi:10.1186/1471-2164-12-110

Cite this article as: Lu et al:: Strain-specific copy number variation in the intelectin locus on the 129 mouse chromosome 1. BMC Genomics 2011 $12: 110$.

\section{Submit your next manuscript to BioMed Central and take full advantage of:}

- Convenient online submission

- Thorough peer review

- No space constraints or color figure charges

- Immediate publication on acceptance

- Inclusion in PubMed, CAS, Scopus and Google Scholar

- Research which is freely available for redistribution

Submit your manuscript at www.biomedcentral.com/submit
C Biomed Central 